\title{
Discrete Laplace Operator in the Space with a Fuzzy Partition
}

\author{
${ }^{*}$ Hana Zámečníkováa ${ }^{a}$ and Irina Perfilieva ${ }^{b}$ \\ ${ }^{a}$ Department of Mathematics, University of Ostrava, Dvořákova 7, Ostrava, 701 03, Czech republic, \\ hana.zamecnikova@osu.cz \\ ${ }^{b}$ Institute for Research and Applications of Fuzzy Modeling, University of Ostrava, Dvořákova 7, Ostrava, \\ 701 03, Czech republic, irina.perfilieva@osu.cz
}

\begin{abstract}
Differential operators definitely play an important role in image processing tasks. One of the most frequently used operator for this purpose is the Laplace operator, usually defined as the divergence of a gradient of a function. Since images are considered as discrete objects, many applications require discrete version of this operator. But at the same time, these discrete Laplace operators should preserve certain properties known from the continuous case. Based on this fact, we present discrete variant of F-transform-based Laplace operator, satisfying such conditions.

Moreover, Laplace operator can be also defined axiaomatically, throughout its certain properties. Therefore, we propose a construction of such operator from the other side, just with notion of the inner product of the current space and its desired properties.
\end{abstract}

Keywords: Discrete Laplace operator, Fuzzy transform, Fuzzy partition, Basic functions, Inner product.

\section{Introduction}

Laplace operator or Laplacian is a second-order differential operator that occurs frequently in the study of field theory. It is defined as the divergence of a gradient

$$
\Delta f=\operatorname{div}(\nabla f)
$$

and therefore maps a scalar function to another scalar function [9].

The significance of this operator is mainly in the fact that at a specific point, it measures how much the average value of function over neighborhood centered at the point differs from true function value at the point [14].

Analogically, the vector (or even tensor) Laplace operator can be defined as a differential operator defined over a vector (resp. tensor) field. The Laplace operator can be generalized to a so-called Laplace-Beltrami operator defined on a Riemannian manifold.

Another generalization of the Laplace operator, pLaplace weighted operator, that occurs in connection to pseudo-Riemannian manifolds, uses the exterior derivative and (with an assumption $p=2$ ) can be expressed as

$$
\Delta f=\frac{1}{2} \mathrm{~d}^{*} \mathrm{~d} f,
$$

where $\mathrm{d}^{*}$ is the adjoint of the derivative [5].

Aforementioned variants of this operator have been presented in a continuous versions. However, in connection with practical applications, a discrete representation is often more appropriate. The discrete Laplace operator is an analog of the continuous Laplace operator [3], commonly called the Laplacian matrix. This matrix can be interpreted as a pointwise representation of a particular case of the discrete Laplace operator [15]. This discrete Laplace operator is incorporated in many applications, especially relating to image processing. Among others, image denoising or segmentation [6] can be mentioned. This approach also turned out to be successful for image colorization [8], dimensionality reduction [2] or clustering [1]. Moreover, Laplacians have been also studied in connection with surface reconstruction problems [13]. Primarily because surface mesh differential properties can be derived from certain Laplace operator defined on the mesh.

It should be mentioned here that each specific application often requires certain structural properties of discrete Laplacians, which leads to a large amount of various discrete versions of this operator. Despite this inconsistent conventional usage, where almost every [15] 
approach selects only the properties, which turn out to be useful and sufficient for its particular study, defining the Laplacian through its properties has several advantages. For instance, it provides nice geometric insight into the character of the operator.

\section{Preliminarities}

Considering image processing, the most important object here is apparently an image. The image is usually represented as set of pixels arranged into certain structure. Alternatively such image can be expressed in the form of a graph, where each pixel is identified with a vertex. Edges of such originated graph then represents similarities between pixels, resulting in a more or less grid structure.

Regarding to this fact, let us recall basic definitions on graphs. Assume a weighted graph $G=(V, E, w)$ with a finite set of vertices $V=\left\{v_{1}, \ldots, v_{n}\right\}$ and a finite set of weighted edges $E(E \subset V \times V)$. Two vertices $u$ and $v$ are connected by an edge $(u, v)$ and a weight of such edge is then $w(u, v)$. The weights are set by using a function $w: V \times V \rightarrow \mathrm{R}_{+}^{0}$, which is symmetric $(w(u, v)=w(v, u), \forall(u, v) \in E)$, non-negative $(w(u, v) \geq 0)$ and $w(u, v)=0$ if $(u, v) \notin E$. Notation $u \sim v$ stands for two adjacent vertices $u$ and $v$, meaning there exists en edge between them.

Note the function $w$ is just one of the possible functions that can be defined on the set $H(E)$.

Let $H(V)$ denote the Hilbert space of real-valued functions on the vertices $V$ of the graph. A function $u: V \rightarrow \mathbb{R}$ of $H(V)$ assigns a real value $u(v)$ to each vertex $v \in V$. The space $H(V)$ contains the usual inner product $\langle f, h\rangle_{H(V)}=\sum_{v \in V} f(v) h(v)$, where $f, h: V \rightarrow \mathbb{R}$. Similarly, let $H(E)$ denote the space of real-valued functions defined on the edges of a graph $G$. This space incorporate the inner product $\langle F, H\rangle_{H(E)}=\sum_{(u, v) \in E} F(u, v) H(u, v)=$ $\sum_{u \in V} \sum_{v \sim u} F(u, v) H(u, v)$, where $F, H: E \rightarrow \mathbb{R}$ are two functions on $H(E)$.

\section{Laplace-Beltrami operator on weighted graphs}

In this section, we recall [5] the definition of Laplace operator in context of Laplace-Beltrami operator, a differential operator given by the divergence of the gradient of a function [5].

Let $G=(V, E, w)$ be a weighted graph, and let $f: V \rightarrow$ $\mathbb{R}$ be a function of $H(V)$. The difference operator of $f$, noted $d: H(V) \rightarrow H(E)$, is defined on an edge $(u, v) \in$
$E$ by

$$
(d f)(u, v)=\sqrt{w(u, v)}(f(v)-f(u)) .
$$

The directional derivative of $f$, at vertex $v \in V$, along the edge $e=(u, v)$, is defined as:

$$
\partial_{v} f(u)=(d f)(u, v) .
$$

The adjoint of the difference operator, noted $d^{*}$ : $H(E) \rightarrow H(V)$, is a linear operator defined by:

$$
\langle d f, H\rangle_{H(E)}=\left\langle f, d^{*} H\right\rangle_{H(V)},
$$

for any functions $H \in H(E)$ and $f \in H(V)$.

Proposition 1: The adjoint operator $d^{*}$ at each vertex $u \in V$ acts as follows:

$$
\left(d^{*} H\right)(u)=\sum_{v \sim u} \sqrt{w(u, v)}(H(v, u)-H(u, v)) .
$$

The divergence operator, opposite to $d^{*}$, measures the network outflow determined by a function in $H(E)$, at each vertex of a graph.

The gradient operator of a function $f \in H(V)$ assigns to each vertex $u \in V, \forall\left(u, v_{i}\right) \in E$, a column vector:

$\nabla_{w} f(u)=\left(\partial_{v} f(u): v \sim u\right)^{T}=\left(\partial_{v_{1}} f(u), \ldots, \partial_{v_{k}} f(u)\right)^{T}$.

Therefore, the Laplace-Beltrami operator of a function $f \in H(V)$, noted $\Delta_{w}: H(V) \rightarrow H(V)$, is identified by:

$$
\Delta_{w} f=-\frac{1}{2} d^{*}(d f) .
$$

For simplicity in following text we will be using just term Laplace operator, keeping in mind that it refers to Laplace-Beltrami operator.

Proposition 2: The Laplace operator of $f \in H(V)$, at a vertex $u \in V$, can be computed by:

$$
\Delta_{w} f(u)=-\sum_{v \sim u} w(u, v)(f(u)-f(v))
$$

This Laplace operator is linear and corresponds to the combinatorial graph Laplacian.

\section{Fuzzy partition and F-transforms}

As we indicated at the beginning, our goal is to contextualize this operator into the theory of Fuzzy transform. In following section, we construct graph representation of discrete space with a fuzzy partition. Below, we connect universe (with the fuzzy partition) with a weighted graph. With this purpose in mind, we now recall the basic notions regarding this topic. 


\subsection{Fuzzy partition}

Definition 1: Fuzzy sets $A_{1}, \ldots, A_{n}$, establish a fuzzy partition of a real interval $[a, b]$ with nodes $x_{1}<\cdots<$ $x_{n}$ if for $k=1, \ldots, n$ holds:

1. $A_{k}:[a, b] \rightarrow[0,1], A_{k}\left(x_{k}\right)=1, A_{k}(x)>0$ if $\quad x \in$ $\left(x_{k-1}, x_{k+1}\right)$

2. $A_{k}(x)=0$ if $x \notin\left(x_{k-1}, x_{k+1}\right)$, where $x_{0}=a$ and $x_{n+1}=b$

3. $A_{k}(x)$ is continuous

4. $A_{k}(x)$, for $k=2, \ldots, n$, strictly increases on $\left[x_{k-1}, x_{k}\right]$ and $A_{k}(x)$ strictly decreases on $\left[x_{k}, x_{k+1}\right]$ for $k=1, \ldots, n-1$,

5. $\forall x \in[a, b]$

$$
\sum_{k=1}^{n} A_{k}(x)=1
$$

The membership functions $A_{1}, \ldots, A_{n}$ are called basic functions [10].

Definition 2: The fuzzy partition $A_{1}, \ldots, A_{n}$, for $n \geq 2$ is $h$-uniform if nodes $x_{0}<\cdots<x_{n+1}$ are h-equidistant, i.e. for all $k=1, \ldots, n+1, x_{k}=x_{k-1}+h$, where $h=$ $(b-a) /(n+1)$ and the following additional properties are fulfilled [10]:

1. for all $k=1, \ldots, n$ and for all $x \in[0, h], A_{k}\left(x_{k}-\right.$ $x)=A_{k}\left(x_{k}+x\right)$,

2. for all $k=2, \ldots, n$ and for all $x \in\left[x_{k-1}, x_{k+1}\right]$, $A_{k}(x)=A_{k-1}(x-h)$.

Definition 3: If the fuzzy partition $A_{1}, \ldots, A_{n}$ of $[a, b]$ is h-uniform, then there exists an even function $A_{0}$ : $[-1,1] \rightarrow[0,1]$, such that for all $k=1, \ldots, n$ :

$$
A_{k}(x)=A_{0}\left(\frac{x-x_{k}}{h}\right), \quad x \in\left[x_{k-1}, x_{k+1}\right] .
$$

$A_{0}$ is called a generating function of uniform fuzzy partition [10].

Corollary 1: Generating function $A_{0}$ produces infinitely many rescaled functions $A_{H}: \mathbb{R} \rightarrow[0,1]$ with the scale factor $H>0$, so that:

$$
A_{H}(x)=A_{0}\left(\frac{x}{H}\right) .
$$

A $(h, H)$-uniform partition of $\mathbb{R}$ is then a collection of translations $\left\{A_{H}(x-k \cdot h), k \in \mathbb{Z}\right\}[11]$.

\subsection{Fuzzy transform}

Direct Fuzzy transform or F-transform is a result of weighted linear integral transformation of a continuous function with weights determined by basic functions.

Definition 4: Let $A_{1}, \ldots, A_{n}$ be basic functions which form a fuzzy partition of $[a, b]$ and $f$ be any function from $C([a, b])$. We say that n-tuple of real numbers $F[f]=\left(F_{1}[f], \ldots, F_{n}[f]\right)$ given by

$$
F_{k}[f]=\frac{\int_{a}^{b} f(x) A_{k}(x) d x}{\int_{a}^{b} A_{k}(x) d x}, \quad k=1, \ldots, n,
$$

is the direct integral F-transform of $f$ with respect to $A_{1}, \ldots, A_{n}[11]$

F-transform establishes a correspondence between a set of continuous functions on $[a, b]$ and the set of n-dimensional vectors. Inverse F-transform then converts an n-dimensional vector of components $\left(F_{1}[f], \ldots, F_{n}[f]\right)$ into another continuous function:

$$
\hat{f}(x)=\sum_{k=1}^{n} F_{k} A_{k}(x),
$$

which approximates the original one [11].

\subsection{Discrete F-transform}

As we mentioned, major part of applications do not consider continuous function as an input. Therefore, let us now modify the previous definition.

Assume that the function $f$ is defined only on a finite set $P=\left\{x_{1}, \ldots, x_{p}\right\} \subseteq[a, b]$.

Definition 5: The domain $P$ of the function $f$ is sufficiently dense with respect to the fixed fuzzy partition if for each fuzzy set $A_{k}, k=1, \ldots, n$, from the fuzzy partition, there is an element $x_{j} \in P$ belonging to the support of $A_{k}$. Formally

$$
(\forall k)(\exists j) A_{k}\left(x_{j}\right)>0 .
$$

Definition 6: Let $A_{1}, \ldots, A_{n}$ be a fuzzy partition of $[a, b]$ and a function $f$ be defined on the set $P=$ $\left\{x_{1}, \ldots, x_{p}\right\} \subseteq[a, b]$ that is sufficiently dense with respect to $A_{1}, \ldots, A_{n}$. We say that the n-tuple of real numbers $F[f]=\left(F_{1}[f], \ldots, F_{n}[f]\right)$ is a discrete F-transform of $f$ with respect to $A_{1}, \ldots, A_{n}$ if

$$
F_{k}[f]=\frac{\sum_{j=1}^{p} f\left(x_{j}\right) A_{k}\left(x_{j}\right)}{\sum_{j=1}^{p} A_{k}\left(x_{j}\right)} .
$$

Note here that according this definition we strictly distinguish points and nodes, meaning not every point from $P$ has to be considered as a node. 


\section{Discrete FT-based Laplacian}

Now we have all necessary tools for introducing the discrete F-transform-based Laplace operator. In this context we propose to identify each point with a node, in contrast with the previously mentioned approach. Let us assume that graph $G=(V, E, w)$ does not contain any self-loops, i.e. $u \nsim u$, and any isolated vertices, meaning graph $G$ is connected. Let weights of edges be defined as follows:

$$
w(u, v)=A_{u}(v)=A(u, v) .
$$

In addition, each weight is multiplied by normalization coefficient. Then, based on equation (6), we can define discrete Laplace operator by its linear action on vertexbased functions:

$$
\begin{aligned}
\Delta f(u) & =\frac{1}{\sum_{v \sim u} A(u, v)} \sum_{v \sim u}(f(v)-f(u)) A(u, v) \\
& =\frac{\sum_{v \sim u} f(v) A(u, v)}{\sum_{v \sim u} A(u, v)}-\frac{\sum_{v \sim u} f(u) A(u, v)}{\sum_{v \sim u} A(u, v)} \\
& =\frac{\sum_{v \sim u} f(v) A(u, v)}{\sum_{v \sim u} A(u, v)}-\frac{f(u) \sum_{v \sim u} A(u, v)}{\sum_{v \sim u} A(u, v)} \\
& =
\end{aligned}
$$

\subsection{Properties}

Discrete Laplacian based on F-transform defined above admits following properties [10]:

1. $\Delta$ vanishes on constant functions.

2. $\Delta$ is symmetric operator, whenever weights are symmetric $(A(u, v)=A(v, u))$.

3. $\Delta$ has a local support. Since weights are defined by basic functions and the latter have local support.

4. Weights are positive, $A(u, v) \geq 0$. Moreover, in a neighbourhood of current vertex $u$, there should always exist such a vertex $v, v \sim u: A(u, v)>0$.

5. Operator $-\Delta$ is positive semi-definite.

6. Linear precision is preserved, $\Delta$ vanishes on all linear functions. $(\Delta f)_{u}=0$ at each interior vertex, whenever $f$ is a linear function on the plane, point-sampled at vertices of $G$.

Proof of this statement is based on analysis of multiple variants of linear functions. To illustrate the idea of the proof, let us assume linear function in the form $f(u)=\alpha u$, and discuss the behavior of Laplacian in the neighbourhood of origin, with generating basic function $A_{0}$.
Then,

$$
\begin{aligned}
F[f]_{i} & =\frac{\sum_{j \sim i} f\left(u_{j}\right) A_{i}\left(u_{j}\right)}{\sum_{j \sim i} A_{i}\left(u_{j}\right)}=\frac{\sum_{j \sim 0} \alpha u_{j} A_{0}\left(u_{j}\right)}{\sum_{j \sim 0} A_{0}\left(u_{j}\right)} \\
& =\frac{\alpha\left(u_{-j} A_{0}\left(u_{-j}\right)+u_{j} A_{0}\left(u_{j}\right)\right)}{A_{0}\left(u_{-j}\right)+A_{0}\left(u_{j}\right)}=\alpha 0=0 .
\end{aligned}
$$

And since $f(0)=0$,

$$
\Delta(f)_{0}=F[f]_{0}-f(0)=0 .
$$

\section{FT-based Laplacian determined by inner product}

Laplace operator can also be constructed using $L^{2}$ inner product and adjoint operator.

For specific properties of chosen inner product (and therefore also Laplacian) we are able to recover particular weights leading us to the definition of respective basic functions and therefore also structure of resulting fuzzy partition.

\subsection{Representation of inner product}

Definition 7: Let $W$ be an $n$-dimensional vector space. An inner product on $W$ is a rule that assigns to each pair $\mathbf{v}, \mathbf{w} \in W$ a real number $(\mathbf{v}, \mathbf{w})$ such that, for all $\mathbf{u}, \mathbf{v}, \mathbf{w} \in V$ and $\alpha \in \mathbb{R}[4]:$

1. $(\mathbf{v}, \mathbf{v}) \geq 0$, with equality if and only if $\mathbf{v}=0$,

2. $(\mathbf{v}, \mathbf{w})=(\mathbf{w}, \mathbf{v})$,

3. $(\mathbf{u}+\mathbf{v}, \mathbf{w})=(\mathbf{u}, \mathbf{w})+(\mathbf{v}, \mathbf{w})$,

4. $(\alpha \mathbf{v}, \mathbf{w})=\alpha(\mathbf{v}, \mathbf{w})$.

Inner product can be alternatively represented in a matrix form. More precisely, if a matrix fulfills the following properties, then it can be associated with appropriate inner product [7].

Theorem 1: Let $W$ be an $n$-dimensional vector space with a basis $B$ and let $A$ be an $n \times n$ symmetric positive definite matrix. Then the formula:

$$
(\mathbf{u}, \mathbf{v})_{A}=\mathbf{x}^{T} A \mathbf{y}
$$

defines an inner product on $W$ [12]. Vectors $\mathbf{u}, \mathbf{v} \in W$, where $\mathbf{x}$ and $\mathbf{y}$ are the coordinate vectors of $\mathbf{u}$ and $\mathbf{v}$, respectively, i.e., $\mathbf{x}=[\mathbf{u}]_{B}$ and $\mathbf{y}=[\mathbf{v}]_{B}$.

In section 5.1 we mentioned certain structural properties of the discrete Laplacian. Based on these properties, let us now define a matrix corresponding to such an inner product. 
Proposition 3: Let $A$ be a square $n \times n$ matrix defined as follows ${ }^{1}$ :

$$
A=\left[a_{i j}\right]=\left\{\begin{array}{c}
a_{i j}=1 \quad \text { if } \quad i=j \\
a_{i j}<1 \\
a_{i j}=a_{j i} \\
a_{i, i-j} \geq a_{i, i-k} \quad \text { if } \quad j \leq k .
\end{array}\right.
$$

A bilinear form determined in accordance with $A$ defines the inner product:

$$
(\mathbf{u}, \mathbf{v})_{A}=\mathbf{x}^{T} A \mathbf{y}=\sum_{j=1}^{n} \sum_{i=1}^{n} x_{i} a_{i j} y_{j}
$$

Corollary 2: Specifically for the Laplace operator on weighted graph we then obtain:

$$
(\Delta f)_{i}:=\left(\Delta f, 1_{i}\right)_{A}=\sum_{j \sim i} \frac{a_{i j}}{\left|e_{i j}\right|^{2}}(f(j)-f(i)),
$$

where $1_{i}$ is a discrete Dirac delta function, which has a unit value at vertex $i$ and vanishes on all others. Assuming equidistantly located vertices with edge weights equal to respective $a_{i j}$ components, we can recover (up to a constant multiple) representation of Laplacian based on discrete fuzzy tranform. Notice that the values of weights defined in (11) fully correspond with values given by the appropriate matrix components

$$
a_{i j}=A(i, j)=A_{i}(j) .
$$

\section{Conclusion}

A discrete representation of Laplace operator in a space with a fuzzy partition was proposed and analysed. It originates from the theory of fuzzy transform, where the weight assignment is based on a generating function of a fuzzy partition and represents proximity between points. We described certain properties for this operator, where each property was motivated by a specific structural property of continuous Laplacian. We also recover this discrete Laplace operator from the other side. Setting desired properties on inner product, resulting in above mentioned definition of basic functions and therefore leading us to specific fuzzy partition.

The next step in our work will be focused on illustration how these established concepts can be incorporated to applications in signal or image processing.

\footnotetext{
${ }^{1}$ The assignment of matrix elements can be considered for instance as:

$$
a_{i j}=1-\frac{|i-j|}{l}
$$

where $l \in \mathrm{N}$. Since $A$ is determined as $n \times n$ matrix, we assume $l<n$.
}

\section{Acknowledgement}

The authors thank the reviewers for their valuable comments and suggestions to improve the quality of the paper. This paper is sponsored by the grant SGS18/PřF$\mathrm{MF} / 2021$.

\section{References}

[1] M. Belkin, P. Niyogi, Laplacian eigenmaps and spectral techniques for embedding and clustering, in: Proceedings of the 14th International Conference on Neural Information Processing Systems: Natural and Synthetic, Vancouver, British Columbia, Canada, 2001, pp. 585-591.

[2] M. Belkin, P. Niyogi, Laplacian eigenmaps for dimensionality reduction and data representation, Neural Computation 15 (2003) 1373-1396.

[3] D. Burago, S. Ivanov, Y. Kurylev, A graph discretization of the laplace-beltrami operator, Journal of Spectral Theory 4 (2014) 675-714.

[4] M. M. Day, Some characterizations of innerproduct spaces, Transactions of the American Mathematical Society 62 (1947) 320-337.

[5] A. Elmoataz, O. Lézoray, S. Bougleux, Discrete regularization on weighted graphs: A framework for image and manifold processing, IEEE Transactions on Image Processing 17 (2008) 10471060 .

[6] G. Gilboa, Nonlocal linear image regularization and supervised segmentation, Multiscale modeling and simulation 6 (2007) 595-630.

[7] D. C. Lay, Linear algebra and its applications, Addison-Wesley, Boston, 2012.

[8] O. Lezoray, V. T. Ta, A. Elmoataz, Nonlocal graph regularization for image colorization, in: 2008 19th International Conference on Pattern Recognition, Tampa, FL, 2008, pp. 1-4.

[9] K. Perera, R. Agarwal, D. O’Regan, Morse Theoretic Aspects of $p$-Laplacian Type Operators, American Mathematical Society, United States of America, 2010.

[10] I. Perfilieva, Fuzzy transforms: Theory and applications, Fuzzy sets and systems 157 (2006) 9931023.

[11] I. Perfilieva, M. Daňková, B. Bede, Towards a higher degree f-transform, Fuzzy sets and systems 180 (2011) 3-19. 
[12] L. Song, H. Lu, Proper inner product with mean displacement for gaussian noise invariant ica, in: Proceedings of The 8th Asian Conference on Machine Learning, Vol. 63, The University of Waikato, Hamilton, New Zealand, 2016, pp. 398-413.

[13] O. Sorkine, Laplacian mesh processing, in: Y. Chrysanthou, M. Magnor (Eds.), Eurographics 2005 - State of the Art Reports, The Eurographics Association, Dublin, 2005, pp. 53-70.

[14] D. Styer, The geometrical significance of the laplacian, American Journal of Physics 83 (2015) 992-997.

[15] M. Wardetzky, S. Mathur, F. Kälberer, E. Grinspun, Discrete laplace operators: No free lunch, in: Proceedings of the Fifth Eurographics Symposium on Geometry Processing, Goslar, DEU, 2007, p. 33-37. 